\title{
CURIE TEMPERATURE AND MAGNETIZATION OF FINITE SUPERLATTICE WITH RANDOM IRREGULARITIES*
}

\author{
W. MaciejeWsiki and W. Reich \\ Institute of Physics, A. Mickiewicz University \\ Matejki 48/49, 60-769 Poznań, Poland
}

\begin{abstract}
The Curie temperature and layer magnetization of ferromagnetic superlattices with random irregularities in modulation amplitude, thickness of layers and width of interdiffusion zones are studied in an extended version of the Kirkwood approximation. It is found that the increase of the deviation in the modulation amplitude gives rise to localized magnetism and the significant increase in the Curie temperature.
\end{abstract}

PACS numbers: $75.70 .-\mathrm{i}$

\section{Introduction}

The Curie temperature $\left(T_{\mathrm{C}}\right)$ of magnetic superlattices formed from alternating thin layers of two ferromagnetic materials has been calculated recently for simple models assuming the perfect, ideally periodic superlattices [1-3], although real structures include random irregularities in the periodicity of compositional modulation which depend on the growth conditions [4].

Hence, it seems advisable to analyse the effect of irregularities in the compositional modulation on the Curie temperature $\left(T_{\mathrm{C}}\right)$ and layer magnetization $\left(\sigma_{p}(T)\right)$ of the ferromagnetic superlattices (FS). The Curie temperature of FS with aperiodic compositional modulation has been calculated very recently in the ordinary molecular field approximation (MFA) [5]. In this paper we study $T_{\mathrm{C}}$ and $\sigma_{p}(T)$ of irregular FS using a generalized mean-field theory which takes partly into account thermal magnetic fluctuations.

*This work was supported by the project 223519102 of the Committee for Scientific Research. 


\section{Model and method}

To describe the ferromagnetic superlattice with random irregularities, we consider the structure as a layered binary alloy $\left(\mathrm{A}_{c} \mathrm{~B}_{1-c}\right)$ of atoms with the spins $S^{\mathrm{A}}$ and $S^{\mathrm{B}}\left(S^{\mathrm{A}}>S^{\mathrm{B}}\right)$. The exchange interaction between the nearest neighbouring spins is described by the exchange integrals $J^{\mathrm{AA}}, J^{\mathrm{BB}}$ and $J^{\mathrm{AB}}\left(J^{\mathrm{AA}}>J^{\mathrm{BB}}\right)$. The concentration of both types of atoms varies around the average value $\left(c^{\text {av }}\right)$ with trapezoidal modulation and therefore, two types of layers can be distinguished: the stronger magnetized layers, rich in atoms $A$ (i.e. with $c_{\mathrm{p}}>c_{\mathrm{av}}$ ) and the weaker magnetized layers, rich in atoms $B$ (i.e. with $c_{\mathrm{p}}<c_{\mathrm{av}}$ ). Interdiffusion is modelled by assuming varying concentrations $c_{\mathrm{p}}$ in the few atomic planes forming the inhomogeneous diffusion zone between the successive layers. The resulting concentration profile across the $n$-th layer $(n=1,2, \ldots, R)$ depends on the amplitude of modulation $\left(F_{n}\right)$, the layer thickness $\left(l_{n}\right)$ and the width of interdiffusion zone $\left(d_{n}\right)$. The irregularities consist in the fluctuations of $F_{n}, l_{n}$ and $d_{n}$ around the average values $F, l$ and $d$. The relative deviations of these parameters are described by

$$
\Delta P_{n}=\left(P_{n} / P\right)-1,
$$

where $P$ denotes $F, l$ or $d$ and the maximum deviation is equal to

$$
\delta P=\max _{n}\left\{\Delta P_{n}\right\}
$$

Hence, the parameters $\delta F, \delta l$ and $\delta d$ determined above can be used as a measure of random irregularities in the modulation amplitude, in the layer thickness, and in the width of the interdiffusion zones, respectively.

To obtain $\sigma_{p}(T)$ and $T_{\mathrm{C}}$ we applied an extended version of the Kirkwood approximation [6] enabling us to develop the mean-field theory to inhomogeneous layered structures. Within such an approach, the layer magnetization in the $p$-th atomic plane of FS can be obtained by solving self-consistently a system of the following $L$ equations:

where

$$
\sigma_{p}=c_{p} \sigma_{p}^{\mathrm{A}}+\left(1-c_{p}\right) \sigma_{p}^{\mathrm{B}}
$$

$$
\sigma_{p}^{\mathrm{A}, \mathrm{B}}=S^{\mathrm{A}, \mathrm{B}} \mathcal{B}\left\{S^{\mathrm{A}, \mathrm{B}}, \frac{S^{\mathrm{A}, \mathrm{B}}}{k T}\left[H_{p}^{\mathrm{A}, \mathrm{B}}-\Delta H_{p}^{\mathrm{A}, \mathrm{B}}\right]\right\}, \quad(p=1,2, \ldots, L) .
$$

$\mathcal{B}\{\ldots\}$ is the Brillouin function, $H_{p}^{\mathrm{A}, \mathrm{B}}$ denotes the effective molecular field acting on the spins from the $p$-th atomic plane and $\Delta H_{p}^{\mathrm{A}, \mathrm{B}}$ is the fluctuation correction term due to the Kirkwood approximation given by

where

$$
\begin{aligned}
& \Delta H_{p}^{\mathrm{A}}=\left(J^{\mathrm{AA}}\right)^{2} \sigma_{p}^{\mathrm{A}}\left[z_{0} c_{p} \Delta \sigma_{p}^{\mathrm{A}}+z_{1} c_{p+1} \Delta \sigma_{p+1}^{\mathrm{A}}+z_{1} c_{p-1} \Delta \sigma_{p-1}^{\mathrm{A}}\right]+\left(J^{\mathrm{AB}}\right)^{2} \\
& \times \sigma_{p}^{\mathrm{A}}\left[z_{0}\left(1-c_{p}\right) \Delta \sigma_{p}^{\mathrm{B}}+z_{1}\left(1-c_{p+1}\right) \Delta \sigma_{p+1}^{\mathrm{B}}+z_{1}\left(1-c_{p-1}\right) \Delta \sigma_{p-1}^{\mathrm{B}}\right], \\
& \Delta H_{p}^{\mathrm{B}}=\left(J^{\mathrm{BB}}\right)^{2} \sigma_{p}^{\mathrm{B}}\left[z_{0}\left(1-c_{p}\right) \Delta \sigma_{p}^{\mathrm{B}}+z_{1}\left(1-c_{p+1}\right) \Delta \sigma_{p+1}^{\mathrm{B}}+z_{1}\left(1-c_{p-1}\right) \Delta \sigma_{p-1}^{\mathrm{B}}\right] \\
& +\left(J^{\mathrm{AB}}\right)^{2} \sigma_{p}^{\mathrm{B}}\left[z_{0} c_{p} \Delta \sigma_{p}^{\mathrm{A}}+z_{1} c_{p+1} \Delta \sigma_{p+1}^{\mathrm{A}}+z_{1} c_{p-1} \Delta \sigma_{p-1}^{\mathrm{A}}\right],
\end{aligned}
$$

$$
\Delta \sigma_{p}^{\mathrm{A}}=\left(S^{\mathrm{A}}\right)^{2}-\left(\sigma_{p}^{\mathrm{A}}\right)^{2},
$$




$$
\Delta \sigma_{p}^{\mathrm{B}}=\left(S^{\mathrm{B}}\right)^{2}-\left(\sigma_{p}^{\mathrm{B}}\right)^{2} .
$$

$z_{0}$ and $z_{1}$ are the numbers of the nearest neighbours in the same layer of FS and in the adjacent layer, respectively.

\section{Numerical results}

We found that the magnetization profiles across FS vary with any perturbation of $F_{n}, l_{n}$ and/or $d_{n}$. Moreover, the sensitivity of $\sigma_{p}(T)$ to the perturbation increases significantly as the temperature increases near $T_{\mathrm{C}}$. This is in contrast with the Curie temperature which, as the calculations imply, is practically insensitive to the perturbation of weakly magnetized layers because $T_{\mathrm{C}}$ of the whole FS is determined by the region which has a higher "local" transition temperature (i.e. is most magnetically enriched).

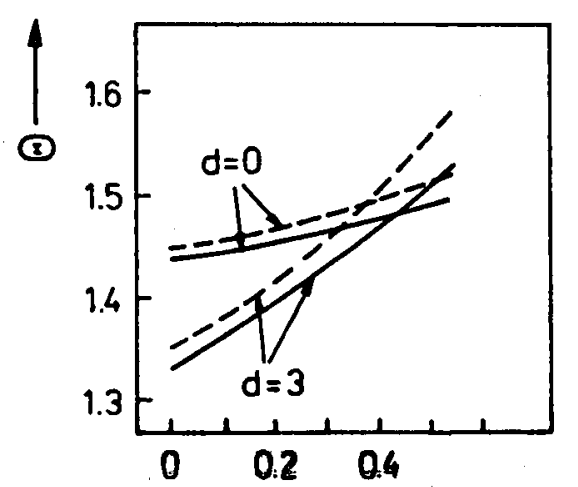

Fig. 1

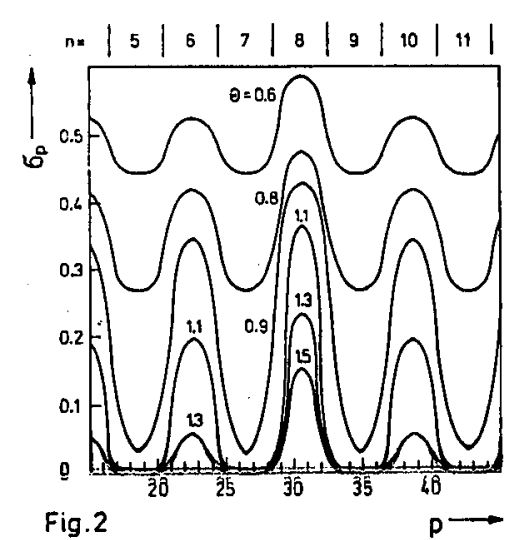

Fig. 1. The influence of the irregularities in the modulation amplitude on the normalized Curie temperature $\Theta$ for different widths of the interdiffusion zones. Solid lines Kirkwood approximation, dashed lines - MFA. The s.c. structure and (001) surface orientation is assumed $\left(S^{\mathrm{A}}=1, S^{\mathrm{B}}=0.5, J^{\mathrm{AA}}=2, J^{\mathrm{BB}}=0.2, J^{\mathrm{AB}}=0.65, F=0.3\right)$; $\Theta=k T_{\mathrm{C}} /\left[J^{\mathrm{AA}} S^{\mathrm{A}}\left(S^{\mathrm{A}}+1\right)+J^{\mathrm{BB}} S^{\mathrm{B}}\left(S^{\mathrm{B}}+1\right)\right]$.

Fig. 2. Magnetization profiles for a superlattice with random irregularities in the modulation amplitude and for various normalized Curie temperatures $\Theta$ attached to each curve. The maximum deviation of the modulation amplitude $\delta F=0.5$. The structural and magnetic parameters are the same as in Fig. 1.

In order to discuss the basic features of the magnetization profiles we select several representative sets of our numerical results. In the following the number of layers in each structure is fixed so that $R=20$ and $l=4$, for a total thickness of $L=80$ atomic planes. Moreover, the s.c. structure and (001) surface orientation are assumed. As seen from Fig. $1, T_{\mathrm{C}}$ significantly increases with $\delta F$ in the case when the source of random irregularities is the fluctuation of modulation amplitude and the increase is higher for FS with thicker interdiffusion zones (greater $d$ ). In 


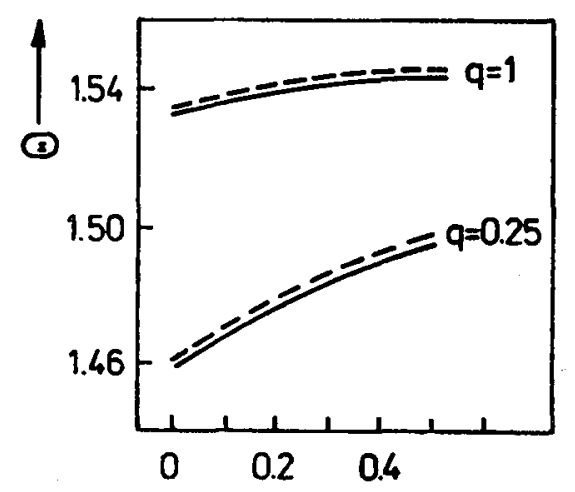

Fig. 3

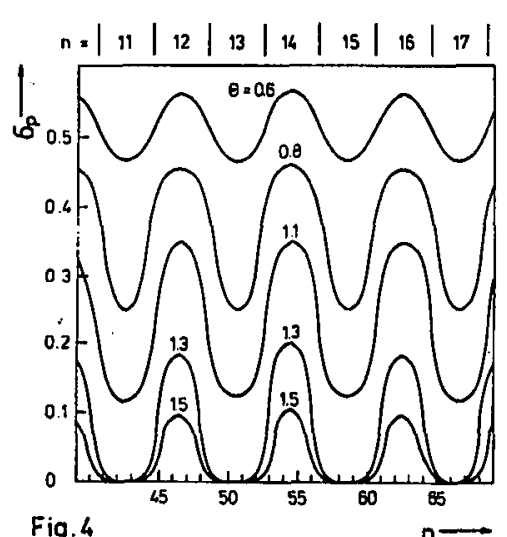

Fig. 4

Fig. 3. The influence of the irregularities in the thickness of layers on the normalized Curie temperature $\Theta$ for different ratios of the a verage thickness of strongly magnetized layers to the average thickness of weakly magnetized ones. Solid lines - Kirkwood approximation, dashed lines - MFA. The structural and magnetic parameters are the same as in Fig. 1

Fig. 4. Magnetization profiles for a superlattice with random irregularities in the thickness of layers and for various normalized Curie temperatures $\Theta$ attached to each curve. The maximum deviation of the thickness of layers $\delta l=0.5$. The structural and magnetic parameters are the same as in Fig. 1.

Fig. 2 the magnetization profiles at various temperatures are shown for the case of $F=0.3$ and $\delta F=0.5$. We present this part of the whole structure which illustrates the cliaracteristle propertics of $\sigma_{p}(T)$. The maximum deviation of the modulation amplitude is assumed to occur in the eighth layer $(n=8)$, i.e. in the atomic planes labelled by $p=29,30,31,32$, which are most magnetically enriched. We see that the largest magnetization appears at the center of this layer and as the temperature increases near $T_{\mathbf{C}}$, only the eighth layer remains to have finite magnetization, whereas other layers of FS become almost paramagnetic. Thus, in the case of random irregularities in the modulation amplitude, the localized magnetism with the localization center on the layer with a maximum value of $F_{n}$ appears in FS.

As regards the influence of the irregularities in layer thicknesses on $T_{\mathrm{C}}$ and $\sigma_{p}(T)$ we found (Fig. 3) that they lead to an increase in $T_{\mathrm{C}}$ only for FS with concentration profile dominated by the weakly magnetized layers $(q<1)$. The magnetization profiles at various temperatures are shown in Fig. 4 for the case of $\delta l=0.5$ and the fourteenth layer as the thickest strongly magnetized layer. We observe that for temperatures significantly lower than $T_{\mathrm{C}}$ the magnetization profiles reflect directly the variation in the chemical composition of successive layers of the structure. However, for temperatures very close to $T_{\mathrm{C}}$ the magnetization of the magnetically weak layers becomes negligible and $\sigma_{p}(T)$ displays the separation of the magnetically strong layers.

The influence of the irregularities in the width of the interdiffusion zones on 
$T_{\mathrm{C}}$ and $\sigma_{p}(T)$ has been also studied numerically. However, quantitative variations of $T_{\mathrm{C}}$ and $\sigma_{p}(T)$ are in this case negligible even for deviation of $\delta d=0.5$.

We conclude suggesting that the increase in the deviation in the modulation amplitude gives rise to localized magnetism and the significant increase in the Curie temperature. Simultaneously, $T_{\mathrm{C}}$ becomes insensitive to finite-size characteristics of the superlattice, i.e. to the thickness and total number of supercells.

\section{References}

[1] H.R. Ma, C.H. Tsai, Solid State' Commun. 55, 499 (1985).

[2] H.K. Sy, Phys. Lett. A 120, 203 (1987).

[3] W. Maciejewski, A. Duda, Solid State Commun. 64, 557 (1987).

[4] P.J. Grundy, M. Ohkoshi, in: Proc. 4th Internat. Conf. on Physics of Magnetic Materials, Eds. W. Gorzkowski, H.K. Lachowicz; H. Szymczak, World Scientific, Singapore 1988, p. 195.

[5] W. Maciejewski, IEEE Trans. Magn. 26, 213 (1990).

[6] T. de Simone, R.M. Stratt, Phys. Rev. B 32, 1537 (1985). 